\title{
Autopercepção corporal de praticantes de exercício físico em academias
}

\author{
Body self-perception of people who perform physical activities at gyms
}

\author{
Diene da Silva Schlickmann ${ }^{1}$, Amna Muhamad Abder Rauf Muhd Ibrahim Hamid ${ }^{1}$, Patrícia Molz², \\ Edna Linhares Garcia ${ }^{1}$, Suzane Beatriz Frantz Krug${ }^{1}$, Silvia Isabel Rech Franke ${ }^{1}$ \\ 1- Universidade de Santa Cruz do Sul - UNISC, Santa Cruz do Sul, RS, Brasil. \\ 2- Pontifícia Universidade Católica do Rio Grande do Sul - PUCRS, Porto Alegre, RS, Brasil.
}

dienedasilva@gmail.com

Palavras-chave:

Imagem Corporal; Autopercepção; Aparência Física; Academia de Ginástica;

Exercício Físico

Keywords:

Body Image; Self Concept; Body Physical Appearance;

Fitness Centers; Exercise

\section{RESUMO}

Introdução: a preocupação com a imagem corporal sofre forte influência de diversos fatores socioculturais, muitas vezes refletindo numa autoavaliação negativa em relação à própria imagem corporal. A constante idealização do corpo perfeito tem sido um dos principais motivos para a realização de exercícios físicos em academias, objetivando a redução do peso corporal acompanhado de aumento da massa muscular. Objetivo: revisar a literatura científica sobre a insatisfação com a imagem corporal, bem como, sobre o método utilizado para sua avaliação em praticantes de exercícios físicos em academias. Método: a presente pesquisa foi realizada em bases de dados cientificas selecionando artigos nos idiomas português, inglês e espanhol, que avaliassem autopercepção corporal e exercícios físicos em praticantes de academia, entre os anos de 2008 a 2018. Resultados: dos 65 estudos selecionados, 55 artigos foram excluídos por não estarem de acordo com o objetivo do estudo, resultando em 10 artigos, que preencheram os critérios de inclusão e foram analisados na íntegra. A prevalência de insatisfação corporal encontrada entre homens e mulheres variou entre $7,8 \%$ a $98,8 \%$, dentre os estudos avaliados, e os métodos utilizados para avaliar a percepção corporal, entre os artigos selecionados, foram o Body Shape Questionnare (BSQ), seguido da Escala de Stunkard. Conclusão: apesar da grande variação, foi verificada uma insatisfação corporal entre praticantes de academia, de ambos os sexos, bastante expressiva. Entretanto, ressalta-se a importância de um instrumento ideal para avaliar a percepção corporal entre os praticantes de academias.

\footnotetext{
ABSTRACT

Introduction: concern with body image is strongly influenced by several sociocultural factors, often reflecting a negative self-assessment of one's body image. The constant idealization of the perfect body has been one of the main reasons for performing physical activities at gyms, aiming at reducing body weight and increasing muscle mass. Objective: to review the scientific literature on body image dissatisfaction, as well as on the method used for its evaluation among people who perform physical activities at gyms. Method: the search was carried out in scientific databases, selecting articles in Portuguese, English and Spanish, which evaluated body self-perception and physical activities in gym users between 2008 and 2018. Results: of the 65 selected studies, 55 were excluded because they were not in agreement with the objective of the study, resulting in 10 articles that met the inclusion criteria and were analyzed in their entirety. The prevalence of body dissatisfaction found between men and women ranged from $7.8 \%$ to $98.8 \%$ among the evaluated studies, and the methods used to evaluate body perception among the selected articles were the Body Shape Questionnaire (BSQ), followed by the Stunkard Scale. Conclusion: despite the great variation, significant body dissatisfaction was found among gym users of both sexes. However, it is worth noting the importance of an ideal instrument to evaluate body perception among gym users.
} 


\section{INTRODUÇÃO}

Imagem corporal é a percepção que o indivíduo tem do próprio corpo, caracterizado por uma representação mental, de tamanho, imagem e forma (estrutura física), expressando sentimentos (positivos ou negativos) associados a tais características, assim como as partes que as constituem. ${ }^{1,2}$ Contudo, ao longo do tempo, o corpo perfeito é ditado pela mídia, no qual perante a sociedade tem se tornado uma referência extremamente compulsiva ${ }^{3}$, no qual a insatisfação corporal tem provocado o desejo de modificar o corpo, conforme os padrões pré-estabelecidos. Muitas vezes, esta insatisfação está relacionada ao desejo de obter o padrão definido de "corpo perfeito", sendo para as mulheres a magreza e para os homens, o corpo mais forte e volumoso. ${ }^{4}$

A prática de exercícios físicos regulares possui inúmeros benefícios para a saúde do individuo, tais como: diminuição da depressão, melhora no bemestar psicológico, bem como a autopercepção do próprio corpo torna-se mais positiva, promovendo benefícios na qualidade de vida do indivíduo tanto no âmbito social, quanto físico e mental. ${ }^{5,6}$ Por outro lado, as distorções na imagem corporal, visando o corpo perfeito, são geralmente acompanhadas da insatisfação corporal, na qual está associada com consequências negativas como transtornos mentais e comportamentos não saudáveis de controle de peso. $^{7}$ Diante dessa situação, a procura por academias para realização de exercícios físicos tem aumentado expressivamente, pois essa tem sido uma estratégia adotada como uma forma de combater à insatisfação com a imagem corporal e, por conseguinte melhorar a autoestima, refletindo, então, em uma maior satisfação com o corpo. ${ }^{6}$

Dessa forma, esse artigo tem por objetivo revisar na literatura científica sobre a insatisfação com a imagem corporal, bem como, sobre o método utilizado para sua avaliação em praticantes de exercícios físicos em academias.

\section{MÉTODO}

Trata-se de um estudo de revisão bibliográfica, de caráter descritivo realizado por meio de artigos científicos publicados nos últimos 10 anos, utilizando, em várias combinações, os seguintes termos de pesquisas: "Imagem Corporal", "Exercício físico" e "Autopercepção Corporal". A pesquisa realizada em bases de dados cientificas: "Scielo", "Pubmed" e "Google acadêmico", incluiu artigos originais publicados nos idiomas português, inglês e espanhol entre os anos de 2008 a 2018, que avaliassem autopercepção corporal em qualquer modalidade de exercícios físicos em praticantes de academia de ambos os sexos.

Foram excluídos artigos de revisão e relato de caso, tese e dissertações. Inicialmente, foram selecionados 65 estudos, após a leitura dos títulos e resumos, foram excluídos 55 artigos que não estavam de acordo com o objetivo do estudo, restando 10 que preencheram os critérios de inclusão, que foram analisados na íntegra e incluídos neste estudo (ver figura 1).

Figura 1. Fluxograma do processo de seleção dos estudos incluídos na revisão.

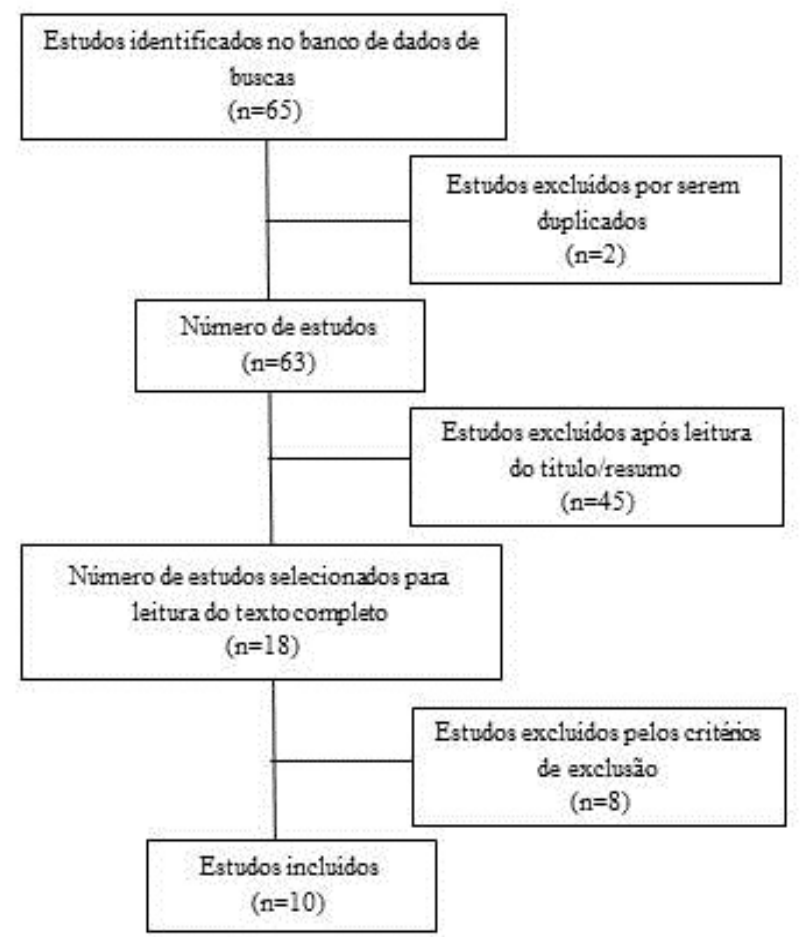

\section{RESULTADOS}

A busca realizada nas bases de dados resultou em 65 artigos, sendo que apenas dez artigos se enquadraram nos critérios de inclusão estabelecidos. Os estudos encontrados apresentaram datas de publicação variando entre 2010 e 2017 e a amostra variou de 31-832 indivíduos. Destes, um artigo avaliou apenas mulheres praticantes de academia, um avaliou homens praticantes de musculação, 
um avaliou universitários de ambos os sexos e o restante, ou seja, sete artigos avaliaram praticantes de academias de ambos os sexos. Ainda, dentre os estudos selecionados, sete artigos usam o questionário Body Shape Questionnare (BSQ) e três a escala de

Tabela 1- Artigos selecionados do ano de 2008 a 2018.
Stunkard para avaliar os indivíduos dos estudos.

As demais informações verificadas nos artigos científicos incluídos na pesquisa estão descritos na tabela 1.

\begin{tabular}{|c|c|c|c|}
\hline Referência & Amostra & Escala Utilizada & Resultados \\
\hline Firmino et al., $2010^{8}$ & $\begin{array}{l}90 \text { indivíduos } \\
(50 \% \text { mulheres })\end{array}$ & Escala Stunkard & $\begin{array}{l}\text { Escore de satisfação corporal foi de } 1,0 \pm 1,2 \text { para as } \\
\text { mulheres e } 0,4 \pm 1,5 \text { para os homens. }\end{array}$ \\
\hline Damasceno et al., $2011^{9}$ & $\begin{array}{l}276 \text { indivíduos } \\
\text { (61\% homens; } 23,9 \pm 5,2\end{array}$ & Escala de Stunkard & $\begin{array}{l}98,8 \% \text { dos homens e } 93,5 \% \text { das mulheres se } \\
\text { consideravam satisfeitas com o corpo. }\end{array}$ \\
\hline Santos et al., $2011^{6}$ & $\begin{array}{c}117 \text { indivíduos } \\
(66 \% \text { mulheres; } 18-32 \text { anos })\end{array}$ & $\begin{array}{c}\text { Body Shape } \\
\text { Questionnare (BSQ) }\end{array}$ & $\begin{array}{l}\text { A classificação do BSQ mostrou que as mulheres } \\
\text { apresentaram uma média de } 80,18 \text { pontos, não } \\
\text { apresentando alteração na percepção da imagem } \\
\text { corporal, porém possuem uma leve tendência } \\
\text { a distorção da imagem corporal. Os homens } \\
\text { apresentaram uma média de } 61,6 \text { pontos, não } \\
\text { apresentando alteração na percepção da imagem } \\
\text { corporal. }\end{array}$ \\
\hline Ferrari et al., $2012^{10}$ & $\begin{array}{l}832 \text { universitários } \\
(58 \% \text { homens; } 20,1 \pm 4,6 \\
\text { anos })\end{array}$ & $\begin{array}{c}\text { Body Shape } \\
\text { Questionnaire (BSQ - } \\
\text { 34) }\end{array}$ & $\begin{array}{l}\text { A prevalência de insatisfação com a imagem corporal } \\
\text { foi de } 10.1 \% \text {, sendo } 11.9 \% \text { entre os homens e } 18.2 \% \\
\text { entre às mulheres. }\end{array}$ \\
\hline Bracht et al. $2013^{7}$ & $\begin{array}{l}31 \text { estudantes } \\
(84 \% \text { mulheres; } \\
23,0 \pm 5,0 \text { anos })\end{array}$ & $\begin{array}{c}\text { Body Shape } \\
\text { Questionnaire (BSQ) }\end{array}$ & $\begin{array}{l}51,7 \% \text { indivíduos presentavam distorção da imagem } \\
\text { corporal }\end{array}$ \\
\hline Silva et al., $2015^{5}$ & $\begin{array}{c}153 \text { indivíduos } \\
\text { (63\% homens; } 17-59 \text { anos) }\end{array}$ & $\begin{array}{c}\text { Body Shape } \\
\text { Questionnaire (BSQ) }\end{array}$ & $7,8 \%$ insatisfação com a imagem corporal \\
\hline Ristow et al., $2013^{11}$ & $\begin{array}{l}284 \text { homens } \\
(26,3 \pm 6,0 \text { anos })\end{array}$ & Esala de Stunkard & $\begin{array}{l}42,3 \% \text { dos praticantes de musculação se encontravam } \\
\text { insatisfeitos com composição }\end{array}$ \\
\hline Costa et al., $2015^{2}$ & $\begin{array}{l}100 \text { indivíduos } \\
(67 \% \text { mulheres })\end{array}$ & $\begin{array}{c}\text { Body Shape } \\
\text { Questionnaire (BSQ) }\end{array}$ & $52 \%$ insatisfação com a imagem corporal \\
\hline Klimick et al., $2017^{3}$ & $\begin{array}{c}120 \text { indivíduos } \\
\text { (50\% homens; } 15-74 \text { anos })\end{array}$ & $\begin{array}{c}\text { Body Shape } \\
\text { Questionnaire (BSQ) }\end{array}$ & $\begin{array}{l}55 \% \text { das mulheres e } 45 \% \text { dos homens possuem algum } \\
\text { nível de insatisfação com a imagem corporal }\end{array}$ \\
\hline Medeiros et al., $2017^{12}$ & $\begin{array}{l}257 \text { mulheres } \\
(18-35 \text { anos })\end{array}$ & $\begin{array}{c}\text { Body Shape } \\
\text { Questionnaire (BSQ) }\end{array}$ & $\begin{array}{l}21,4 \% \text { indivíduos apresentaram insatisfação com a } \\
\text { imagem corporal }\end{array}$ \\
\hline
\end{tabular}

\section{DISCUSSÃO}

Nos últimos anos tem se observado maior preocupação com a imagem corporal em todas as faixas etárias. ${ }^{13,14}$ Com isso, as academias têm ganhado mais adeptos que visam utilizar a prática de exercícios físicos para obter um corpo adequado aos padrões culturalmente impostos. ${ }^{5-15}$

Dentre os artigos pesquisados, a prevalência de insatisfação corporal variou entre 7,8\% a 98,8\%, avaliando tanto homens, quanto mulheres e para a avaliação da imagem corporal, os instrumentos mais utilizados têm sido as escalas de silhuetas, também conhecidas como contour line drawing ou figural drawing. ${ }^{2,3,5-7,9-12}$ Essa escalas são representadas com figuras que geralmente variam de um sujeito muito magro a um obeso, e, o indivíduo entrevistado deve escolher qual figura melhor o representa (silhueta atual) e com qual gostaria de se parecer (silhueta desejada). A partir destes dados a insatisfação corporal é avaliada pela discrepância entre essas medidas. ${ }^{16}$

Em relação à imagem corporal, evidências têm mostrado que as mulheres estão mais preocupadas com um corpo magro e mais "delgado", diferentemente dos homens que buscam um corpo mais volumoso com o aumento de massa muscular ${ }^{5}$. Não obstante a isso, entre os estudos selecionados, a maioria deles indicam uma maior distorção da imagem corporal 
entre as mulheres em relação aos homens. ${ }^{2-5,6}$ Uma possível explicação para tal achado é que homens apresentam maior insatisfação por magreza e, por outro lado, as escalas utilizadas para a avaliação da insatisfação corporal (por exemplo, a BSQ) avaliam a insatisfação corporal apenas para excesso de peso, o que poderia estar influenciando nos resultados encontrados. ${ }^{10}$

$\mathrm{Na}$ pesquisa de Damasceno e colaboradores ${ }^{9}$ pode-se verificar que, muitas vezes, a relação entre composição corporal e insatisfação corporal ocorre somente entre os indivíduos que se sentem muitos insatisfeitos com sua imagem corporal e aparência. A insatisfação corporal tem sido associada muito mais ao bem estar físico que à condição física. ${ }^{8}$

Contudo, para praticantes de academia não existe uma escala estabelecida para avaliação de insatisfação corporal especificamente destes indivíduos ${ }^{2}$. Entretanto, nos estudos selecionados, as escalas mais utilizadas foram a Body Shape Questionnaire (BSQ) $)^{2,3,5-7,10-12}$ e a escala de Stunkard. ${ }^{8,9-11}$ A escala BSQ é composta por um questionário com 34 itens auto preenchíveis e tem por objetivo mensurar a insatisfação corporal através de massa corporal obtida através de somatórios de cada questão, sendo que a pontuação abaixo de 110 não apresenta insatisfação; considera-se insatisfação leve para valores entre 111 a 138; somatório de 139 a 167 indicam insatisfação moderada; e igual ou maior que 168 indicam grave insatisfação corporal. ${ }^{17}$ Já a Escala de Stunkard é um instrumento constituído por uma sequência de nove figuras de corpos femininos ou masculinos, representando indivíduos de diferentes tamanhos corporais, do mais magro ao mais obeso. ${ }^{18}$

Entretanto, Damasceno e colaboradores 9 , ressaltam que as escalas de silhuetas utilizadas para a avaliação de praticantes de exercícios físicos em academia são insuficientes para estudar insatisfação corporal nestes indivíduos, uma vez que os indivíduos visam aumentar a musculosidade e diminuir a gordura corporal. Ademais, Ferrari e colaboradores ${ }^{10}$ indicam que o uso da escala de silhuetas bidimensionais, entre praticantes de exercícios físicos, não permite a representação total do indivíduo, da distribuição de sua massa de gordura e de outros aspectos antropométricos importantes na formação da imagem corporal. Essas escalas normalmente utilizadas nestes estudos, na realidade, são mais adequadas para avaliação de distúrbios alimentares relacionados à imagem corporal.
Ademais, a insatisfação corporal pode ser associada a pensamentos e sentimentos negativos, e a falha na autorregulação de exercícios que envolvem mudança de um estado presente para um desejado. Contudo, essa preocupação excessiva com o corpo pode levar o individuo a ter um reflexo negativo da sua imagem corporal, gerando um desequilíbrio e depreciação das características físicas, como tamanho, forma, peso e massa muscular, bem como alterações no desempenho psicossocial, diminuindo a autoestima, afetando diretamente sua vida pessoal e social. ${ }^{11}$

Com isso, a insatisfação corporal tende estar associado a um padrão excessivo da prática de exercícios físicos. Em relação a isso, tem-se notado que as mulheres tendem a se exercitar-se mais a fim de controlar o peso, tonificar o corpo e melhorar a aparência. $^{6}$

Se por um lado a autoestima está intimamente associada ao bem estar físico e mental, o exercício físico pode contribuir para melhorar a auto estima, pois auxilia na modulação das formas corporais. ${ }^{6}$ Desta forma, a insatisfação corporal surge com a crença de estar obeso, apesar do indivíduo estar com peso adequado e, entre as mulheres, essa distorção muitas vezes ainda pode se associar a distúrbios alimentares, prejudicando a saúde das mesmas. ${ }^{7}$

\section{CONCLUSÃO}

Fica evidente nessa pesquisa a insatisfação corporal entre homens e mulheres praticantes de exercícios físicos em academias. Geralmente esse público busca metas de imagem corporal com base na perda de peso e aumento de massa muscular, buscando desenvolver medidas antropométricas consideradas ideais. Indivíduos que estão insatisfeitos com seus corpos correm maior risco de autoavaliações negativas quando expostos a imagens idealizadas. Nesse sentido, uma percepção errônea da imagem corporal pode comprometer a saúde física e mental desses indivíduos. No entanto, esse estudo deixa lacunas importantes sobre a adequação de uma escala de silhuetas para praticantes de exercícios físicos em academias.

\section{REFERÊNCIAS}

1. Bandeira YE, Mendes AL, Cavalcante AC, Arruda SP. Avaliação da imagem corporal de estudantes do curso de Nutrição de 
um centro universitário particular de Fortaleza. J Bras Psiquiatr 2016;65(2):168-73. doi: http://dx.doi.org/10.1590/00472085000000119

2. Costa AC, Torre MC, Alvarenga MD. Attitudes towards physical exercise and body image dissatisfaction of individuals who frequently go to gyms. Revista Brasileira de Educação Física e Esporte 2015;29(3):453-64. doi: http://dx.doi.org/10.1590/180755092015000300453

3. Klimick AC, Coelho CE, Alli-Feldmann LR. Nível de satisfação corporal de praticantes de musculação em academias de Porto Alegre, RS. Revista de Iniciação Científica da ULBRA 2017;1(15).

4. Tod D, Hall G, Edwards C. Gender invariance and correlates of the Drive for Leanness Scale. Body Image 2012;9(4):555-8. doi: http://dx.doi.org/10.1016/j.bodyim.2012.06.004

5. Silva RS, Ferreira VL. Avaliação da auto-imagem em frequentadores de academia no interior do Rio Grande do Sul. Encontro: Revista de psicologia 2015;16(24):23-30.

6. dos Santos, A.P.R., da Silva, F.J. and Romanholo, R.A. Distúrbios de imagem corporal e sua associação ao nível de atividade física em praticantes de musculação na cidade de Cacoal-RO. Revista Brasileira de Prescrição e Fisiologia do Exercício 2011;5(25):45-50.

7. Bracht CM, Piasetzki CT, Busnello MB, Berlezi EM, Franz LB, Boff ET. Percepção da autoimagem corporal, estado nutricional e prática de atividade física de universitários do Rio Grande do Sul. Mundo Saúde 2013;37(3):343-53.

8. Fermino RC, Pezzini MR, Reis RS. Motivos para prática de atividade física e imagem corporal em frequentadores de academia. Revista Brasileira de Medicina do Esporte 2010;16(1):1823. doi: http://dx.doi.org/10.1590/S1517-86922010000100003

9. Oliveira Damasceno V, Macedo Vianna J, Silva Novaes J, Perrout de Lima J, Fernandes HM, Machado Reis V. Relationship between anthropometric variables and body image dissatisfaction among fitness center users. Revista de Psicología del deporte 2011;20(2):367-82.

10. Ferrari EP, Gordia AP, Martins CR, Silva DA, Quadros TM, Petroski EL. Insatisfação com a imagem corporal e relação com o nível de atividade física e estado nutricional em universitários. Motri 2012;8(3):52-8. doi: http://dx.doi.org/10.6063/motricidade.8(3). 1156

11. Ristow M, dos Santos L, Beims DF, Nesello LÂ. Percepção corporal por praticantes de musculação. Revista da UNIFEBE 2013;1(11).

12. Medeiros TH, Caputo EL, Domingues MR. Insatisfação corporal em frequentadoras de academia. J Bras Psiquiatr 2017;66(1):38-44. doi: http://dx.doi.org/10.1590/00472085000000148

13. Revuelta L, Esnaola I, Goñi A. Relaciones entre el autoconcepto físico y la actividad físico-deportiva adolescente. Revista internacional de Medicina y Ciencias de la Actividad Física del Deporte 2016. doi: http://dx.doi.org/10.15366/rimcafd2016.63.010

14. Souto SV, Novaes JD, Monteiro MD, Neto GR, Carvalhal
MI, Coelho E. Imagem corporal em mulheres adultas vs. meia-idade e idosas praticantes e não praticantes de hidroginástica. Motri 2016;12(1):53-9. doi: http://dx.doi.org/10.6063/motricidade. 5000

15. Ferreira KP, Berleze KJ, Gallon CW. Antropometria, alimentação e auto-imagem corporal de mulheres frequentadoras de academia de Caxias do Sul-RS. Revista Brasileira de Nutrição Esportiva. 2011;5(29):8.

16. Côrtes MG, Meireles AL, Friche AA, Caiaffa WT, Xavier CC. O uso de escalas de silhuetas na avaliação da satisfação corporal de adolescentes: revisão sistemática da literatura. Cad Saude Publica 2013;29:427-44. doi: http://dx.doi.org/10.1590/ S0102-311X2013000300003

17. Carvalho PH, Filgueiras JF, Neves CM, Coelho FD, Ferreira ME. Checagem corporal, atitude alimentar inadequada e insatisfação com a imagem corporal de jovens universitários. J Bras Psiquiatr 2013;62(2):108-14. doi: http://dx.doi.org/10.1590/ S0047-20852013000200003

18. Stunkard AJ, Sørensen T, Schulsinger F. Use of the Danish Adoption Register for the study of obesity and thinness. Res Publ Assoc Res Nerv Ment Dis 1983; 60:115-20.

Como citar: SCHLICKMANN, Diene da Silva et al. Autopercepção corporal de praticantes de exercício físico em academias. Revista Interdisciplinar de Promoção da Saúde, Santa Cruz do Sul, v. 1, n. 3, jul. 2018. ISSN 2595-3664. Disponível em: <https://online.unisc.br/seer/index.php/ripsunisc/article/view/12952>. Acesso em: 22 dez. 2018. doi:https://doi.org/10.17058/rips. v1i3.12952 